\title{
Can a dog be spontaneous?
}

\section{Clive D. L. Wynne ${ }^{1}$ (D)}

Published online: 6 July 2020

(C) The Psychonomic Society, Inc. 2020

\section{Summary}

Fugazza and Miklósi (Scientific Reports, 10(1), 3082, 2020) reported "spontaneous" categorization in a dog. I explore what it means to claim that forms of animal cognition are spontaneous.

Keywords Categorization $\cdot$ Cognitive ethology $\cdot$ Comparative cognition $\cdot$ Concept learning $\cdot$ Discrimination

"Spontaneity is only a term for man's ignorance of the gods."

-Samuel Butler, Erewhon, 1872, p. 262

Fugazza and Miklósi (2020) published a paper with the stimulating title, "Depths and Limits of Spontaneous Categorization in a Family Dog." In this commentary, I focus on just one of the highly thought-provoking concepts raised in this paper: "spontaneous."

What does it mean to claim a behavior is spontaneous, and why should it matter whether the categorization behavior shown by an animal qualifies as spontaneous? The answer surely lies in the recognition that animals, especially biddable animals like dogs, can be trained to do many things, but any credit in that performance accrues to the person who devised and carried out the training, not the animal itself. There is a world of difference between a dog performing tricks and an animal that carries out ingenious acts without requiring human instruction. A recent example from our own work illustrates this point. Van Bourg, Patterson, and Wynne (2020) reported on the propensity of dogs to open a box to free their apparently distressed owner. Approximately one dog in three successfully completed this task within the 2 minutes. To any dog trainer, this level of success appears shockingly low: the task is very simple; surely most dogs could be quickly trained to open a large box. The purpose of our study, however, was to assess the dogs' motivation to aid their human in the absence of any

Clive D. L. Wynne

clivewynne@gmail.com

1 Department of Psychology, Arizona State University, PO Box 871104, Tempe, AZ 85281, USA explicit training. Consequently, spontaneity of the behavior was an essential aspect of what we were studying.

Discussion of spontaneous behavior connects to a deep theme in the history of the study of animal behavior. Animal behavior in general, and the study of animal cognition in particular, is the product of a split and intertwined history.

For most of the 20th century, in continental Europe, the ethological school reigned. This approach to animal behavior was founded by Niko Tinbergen (1907-1988) and Konrad Lorenz (1903-1989), and it emphasized the inborn, inherited, innate behavior of animals. Concurrently, in North America, behaviorism evolved into the behavior analysis of B. F. Skinner (1904 1990). There was a sense for Skinner in which all behavior was spontaneous, but he argued that most of the behaviors he investigated were a function of their environment. This would also apply to the complex patterns of behavior commonly labelled "cognitive" that Skinner eschewed. Environment could include not just the immediate context, but an individual's lifetime history of experiences, and even the evolutionary history that precedes an individual's existence. In practice, behavior analysis emphasized the contingent nature of behavior, predicated on preceding conditions and consequences.

Although the founders of ethology and behavior analysis are long gone, the divide between the predominantly phylogenetic explanations coming from the ethological tradition and the ontogenetic explanations of the behavior analysists continues to have ramifications for scientists studying animal behavior today.

Fugazza and Miklósi acknowledge that prior studies have demonstrated "categorization . . . after extensive training procedures under laboratory conditions" (Fugazza \& Miklósi, 2020 , p. 1), but claim originality in their report because their dog was not trained; its categorization behavior was spontaneous. 
Categorization is the ability to treat classes of objects that are clearly different as being functionally equivalent (Wasserman, 2016). A category can be based around perceptual similarity. A category can also be functional—meaning that objects are treated as equivalent because they are associated with similar actions, lead to similar outcomes or functions, or for some other, possibly arbitrary reason (Wasserman, 2016). The dog subject, Whisky, that Fugazza and Miklósi (2020) report upon, had been trained by a family in Norway to collect on command 59 objects identified by vocal labels. Among these objects, the dog had been trained to recognize four categories: balls, Frisbees, rings, and ropes. The vocal labels for each object in these four classes included the label for each class: thus, one Frisbee was labelled "the small Frisbee"; one of the ropes was "the colorful rope," and so on. In addition to having labels that included as their terminal sound the label for the category as a whole ("ball," etc.), each category was also associated with a different consequence. The trainer engaged the dog in play with objects from each category in a distinctive way (Fugazza \& Miklósi, 2020).

First, Fugazza and Miklósi confirmed that the dog would retrieve an object from a mixed group on the command "bring $<$ name of object>" (p. 7). Each of 59 toys was requested once, and the dog achieved 54 out of 59 correct. Next, the dog was exposed to novel objects from each of the four categories and requested to retrieve the novel object from a collection of four novel objects, one from each category, with the command "bring < name of category>" - for example, "bring Frisbee."

This experiment included two conditions. In one condition the dog was preexposed to the novel objects without any form of human interaction - neither play nor vocal labelling. When tested, the dog performed correctly on 17 of 44 trials. Since there were four objects, chance performance is 11 of 44 trials. This result is reported as significant on a one-tailed binomial test, but would not have been significant on a two-tailed test.

In the other condition, the owner played with the dog and the toy "in the typical way he would play with that type of toy" (p. 4). In the test following this active exposure condition, the dog achieved 24 of 44 trials correct, which is significantly above chance on a two-tailed binomial test and also a significantly higher level of performance than in the first mereexposure condition.

Fugazza and Miklósi (2020) specify that the dog "was not trained specifically for categorization" (p. 4) and repeatedlyin the title and throughout the paper-use the term "spontaneous" to describe its behavior. At one level of analysis, this claim has to be taken seriously: It seems unlikely that the Norwegian family who owned this dog had any explicit understanding of how animals should be trained to form categories. But this is surely a trivial kind of spontaneity. At another level of analysis, the claim is patently absurd: Who would imagine that a dog without any experience of balls, Frisbees, ropes, and rings, the different things people might do with them, and the different vocal labels that people might apply to them, could possible know to respond to them differentially? Without suitable experience, why and how would the dog respond at all to "bring ball," or differentially to that instruction and "bring Frisbee?" The significant difference in performance between the condition where the novel toy was played with compared with the condition where the novel object was simply presented to the dog already indicates the importance of experience of characteristic outcomes for successful categorization. The hint of categorization success, even without differential play opportunities, suggests that some of the categorization may follow from distinctive perceptual qualities of the objects in the different categories, such as smell, taste, shape, and texture.

Without the assertion of spontaneity, the paper loses its claim of originality (though the level of training for categorization to which this dog was exposed is, in any case, very similar to that in Pilley \& Reid, 2011, and Feuerbacher and Rosales-Ruiz, 2017; and categorization can also be identified in dogs trained in olfactory detection work), but, more importantly, the focus of the study shifts from identifying a behavior of the class that could be innate (phylogenetically driven) to one that is acquired (ontogenetically driven).

Surely, 30 years after the deaths of the founders of the ethological/behavior analytic split in animal behavior studies this crude dichotomy can be sustained no longer. In principle, there are no innate behaviors, just as there are no behaviors that are solely the consequence of individual experience. The more complex a behavior is, the more it must be dependent on particular and peculiar patterns of experience. To call Whisky's categorization behavior "spontaneous" is to ignore the exceptional and extended patterns of structured interactions with humans that led to this dog joining the tiny minority of dogs that can respond appropriately to more than a handful of human words. To call this pattern of behavior spontaneous is, to paraphrase and extend Samuel Butler, to be ignorant of the gods of biology.

\section{References}

Feuerbacher, E. N., \& Rosales-Ruiz, J. (2017). Can dogs learn concepts the same way we do? Concept formation in a German Shepherd. International Journal of Comparative Psychology, 30, 1-28.

Fugazza, C., \& Miklósi, Á. (2020). Depths and limits of spontaneous categorization in a family dog. Scientific Reports, 10(1), 3082. doi: https://doi.org/10.1038/s41598-020-59965-6

Pilley, J. W., \& Reid, A. K. (2011). Border collie comprehends object names as verbal referents. Behavioural Processes, 86(2), 184-195.

Van Bourg, J. V., Patterson, J. E., \& Wynne, C. D. L. (2020). Pet dogs (Canis lupus familiaris) release their trapped and distressed owners: Individual variation and evidence of emotional contagion. PLOS ONE, 15(4), e0231742. doi:https://doi.org/10.1371/journal.pone.0231742

Wasserman, E. A. (2016). Conceptualization in pigeons: The evolution of a paradigm. Comparative Cognition: In Honor of Ed Wasserman, $123,4-14$.

Publisher's note Springer Nature remains neutral with regard to jurisdictional claims in published maps and institutional affiliations. 